\title{
THE IMPACT OF TOOTHBRUSH FILAMENT DESIGN ON GINGIVAL HEALTH DURING HEALING. A RANDOMIZED, CONTROLLED, INVESTIGATOR-BLINDED CLINICAL TRIAL
}

\author{
Eva Koval'ová ${ }^{1}$, Bohuslav Novák ${ }^{2}$, Tatiana Klamárová ${ }^{1}$, Anna Eliašová ${ }^{1}$ \\ ${ }^{1}$ Clinic of Maxillofacial Surgery; Faculty of Health Care, University of Prešov in Prešov \\ ${ }^{2}$ Department of Stomatology \& Maxillofacial Surgery FM CU and OISE, Bratislava
}

Kovalová E., Novák B., Klamárová T., Eliašová A. (2015), The impact of toothbrush filament design on gingival health during healing. A randomized, controlled, investigator-blinded clinical trial. Health Problems of Civilization, 1 (9), p. 47-53

\begin{abstract}
Summary:
Objectives. The primary objective of the study was to test toothbrushes with different types of filaments (conical vs. rounded) with respect to cause gingival abrasion after surgical intervention of wisdom teeth. A secondary objective was to evaluate the efficacy of plaque removal and the improvement of gingival conditions to alleviate wound healing and to avoid gingivitis.

Methods. One hundred and seventy-three healthy subjects with surgical intervention of wisdom teeth participated in a randomized, single blind study and were randomly allocated to control group (standard ADA reference toothbrush) or test group (meridol ${ }^{\circledR}$ special toothbrush with conical filaments). Clinical examinations included gingival abrasion, plaque index and gingival index, and were conducted at baseline, 7 and 28 days.

Results. For the gingival abrasion the mean number of lesions of all sizes was after 28 days significantly lower in the test group ( $p$-value <0.001) compared to control group. Plaque index was not significantly different between the two groups in the last visit. At day 28 the gingival index was significantly lower in the test group ( $\mathrm{p}=0.031)$ compared to control group.

Conclusions. The toothbrush with conical filaments induced significantly less gingival abrasions than the standard ADA toothbrush and showed superior results in improving gingival health (gingival index). Both toothbrushes were comparable effective with respect to plaque removal.

Clinical Relevance.

Scientific rationale for study: Supra and sub-gingival biofilm leads to gingival inflammation. Post-surgical removal of the biofilm from gingival surfaces promotes healing after wisdom tooth extraction. Tooth brushing leads to gingival abrasion. Earlier investigations with toothbrushes having conical filaments suggest less gingival tissue damage.

Principal findings: The results showed that the toothbrush with conical filaments caused significantly less gingival abrasions than the toothbrush with rounded filaments.

Practical implications: Toothbrush filament design should be considered when choosing toothbrush for oral hygiene after oral surgery.
\end{abstract}

Keywords: conical filaments, toothbrush, gingival health, gingival abrasion, healing

\section{Introduction}

Gingivitis is caused by substances derived from microbial plaque (biofilm) accumulating at or near the gingival sulcus. All other suspected local and systemic etiologic factors either enhance plaque accumulation or retention, or enhance the susceptibility of the gingival tissue to microbial attack. The evidence supports the conclusion that gingivitis is a disease, and that control and prevention is a worthwhile goal and a health benefit (Page, Gingivitis 1986). Following surgical intervention, the healing process invokes an inflammatory response and, subsequently, the presence of inflammation promotes the rapid formation of a biofilm. It has been shown that significantly more biofilm is formed in the presence of experimentally induced gingivitis than in a healthy environment (Dal. Highfield 1996). After dental extraction, in order to promote wound healing and to avoid gingival damage, the formation of this biofilm should be controlled, both mechanically and chemically, as a combined approach leads to optimal results. Toothbrushes remove biofilm and food debris and thus contribute to reducing inflammation (Saxer, Yankell 1997). Characteristics of an ideal toothbrush would therefore include reliable plaque removal and avoidance of gingival damage due to mechanical abrasion. These properties can be achieved by exploiting properties of the

Address for correspondence: Eva Kovalová, Clinic of Maxillofacial Surgery, Faculty Hospital, Hollého 14, 08001 Prešov, Slovakia, e-mail: kovalova@nextra.sk, phone: +421908974 703

Tables: 2 Figures: 3 References: 16 Full-text PDF www.hpc.edu.pl Copyright (C) Pope John Paul II State School of Higher Education in Biała Podlaska, Sidorska 95/97, 21-500 Biała Podlaska Indexation: Index Copernicus, Database AGRO, ProQuest, Polish Ministry of Science and Higher Education. This is an open-access article distributed under the terms of the Creative Common Attribution Non-commercial license (http://creativecommons.org/licenses/by-nc/3.0), which permits use, distribution and reproduction in any medium, provided the original works is properly cited, the use is non-commercial and is otherwise in compliance with the license. 
filaments and the shape of the inserted handle segment (Drisko et al. 1995). Research that compared the tapered filament toothbrush to a ADA reference toothbrush has revealed that the two types were comparably efficacious in reducing gingivitis, while the toothbrush with tapered filaments was superior in preventing gingival lesions (von Bethlenfalvy et al. 2002 ). Furthermore, with respect to the Turesky plaque score (derived from the QuigleyHein index), there was again a small but significant advantage of the tapered toothbrush (Dörfer et al. 2003). In a split-mouth design trial including 32 subjects, Versteeg et al. compared the tapered filament toothbrush to an ADA reference toothbrush. Here the focus was on gingival abrasion and gingival health following experimental gingivitis. Results showed that, while gingival health improved with both toothbrushes and the tapered filament toothbrush showed a tendency towards fewer gingival sites with abrasion, the ADA toothbrush was more effective in removing plaque and reducing bleeding (Versteeg et al. 2008). In a long-term trial lasting 6 months with 2x15 volunteers, the tapered filament toothbrush was again compared to an ADA reference toothbrush by Checchi et al. (2007). While both the gingival index and the plaque index were significantly improved during treatment in both groups, there were, in comparison, statistically significant advantages with the tapered bristles.

The present study's purpose was to test toothbrushes with different types of filaments (conical vs. rounded) with respect to cause gingival abrasion after surgical intervention of wisdom teeth and to evaluate the efficacy of plaque removal and the improvement of gingival conditions to alleviate wound healing and to avoid gingivitis.

\section{Materials and methods}

\section{Study design and materials}

The study was designed as an investigator-blinded, randomized, controlled trial with two parallel treatment arms. The subjects (healthy individuals aged 18-65) were recruited among patients undergoing surgical extraction of wisdom teeth at the Clinic for Maxillofacial Surgery and Dental Hygiene, Prešov, Slovakia. Study interventions took place between October 2011 and June 2012. The trial was approved by the local ethical committee in Prešov. All patients were informed about the study protocol and signed an informed consent form before beginning the study.

Inclusion criteria were as follows:

- healthy male and female adults aged 18-65 years,

- directly involved after the surgery intervention,

- written informed consent,

- acceptable compliance according to the investigators assessment.

Exclusion criteria were as follows:

- medication that may interfere with the study up to 3 months before study start and during the course of the study,

- allergies and idiosyncratic responses to study products' ingredients,

- teeth supporting structures with any other painful pathology or defects (rampant caries, cracked enamel...),

- systemic diseases,

- known pregnancy or breast-feeding during the course of the study,

- professional administration and home use of CHX or other antibacterial activity mouth rinses or toothpastes,

- fillings etc. within 4 weeks prior enrolment and during the whole study,

- participation in another clinical trial or receipt of an investigational compound/treatment at the same time and 4 weeks prior to inclusion.

The 173 subjects, of whom 74 (42.8\%) were males and 99 (57.2\%) were females, were randomized into the trial's two treatment arms as follows:

Test group - toothbrush with conical filaments (meridol ${ }^{\circledR}$ special toothbrush - Figure 1): 84 patients (32 males, 52 females). 


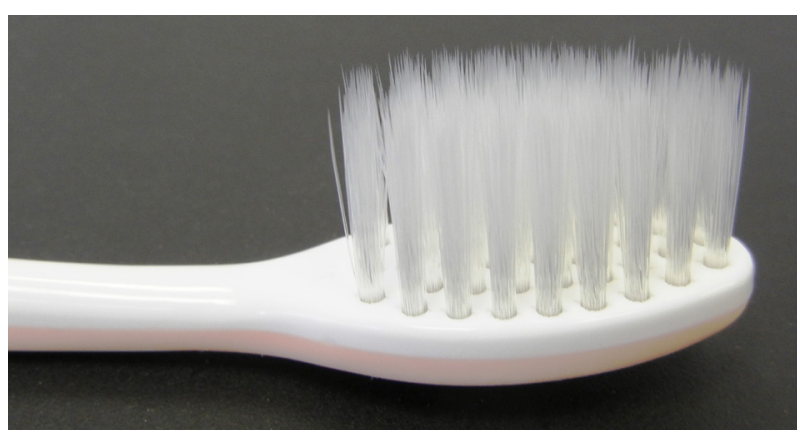

Figure 1. Meridol ${ }^{\circledR}$ special toothbrush with conical filaments

Control group - toothbrush with rounded filaments (ADA reference toothbrush - Figure 2): 89 patients (42 males, 47 females).

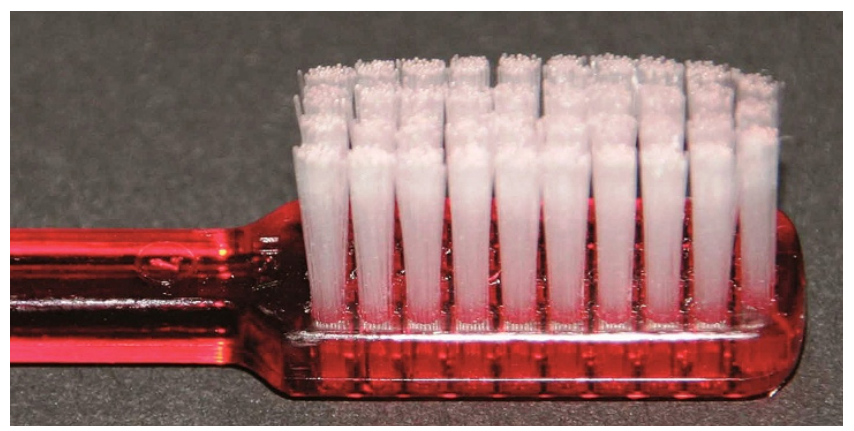

Figure 2. ADA reference toothbrush with rounded filaments

The study was performed under investigator-blinded conditions. Until the final examination of the last participant, the knowledge of the randomization list was limited to the persons responsible for their creation (statistician). In particular, the investigators had no knowledge of the treatment assignments until the blind data review meeting on June 4, 2012. At screening, participants were assigned to consecutive numbers (subject number) according to chronological entry in the study, starting at 20. All study participants were given the assigned toothbrush in anonymous packaging, same toothpaste (meridol ${ }^{\circledR}$ toothpaste) and same mouth rinse (meridol ${ }^{\circledR}$ ). Toothbrush with conical filaments, toothpaste and mouth rinse were provided by the manufacturer. The subjects were instructed to brush their teeth twice daily and rinse in the evening with $10 \mathrm{ml}$ mouth rinse.

\section{Clinical evaluation}

The gingival abrasion was assessed at baseline, day 7, and day 28. In order to visualize gingival abrasion and plaque for assessment, a commercially available staining agent, Mira-2-Ton ${ }^{\circledR}$ solution (Hager Werken, Duisburg/ Germany), was applied to the gums and dental surfaces. This preparation, which is marketed for the visualization of plaque, highlights abraded areas in a different color than intact mucosa.

Gingival tissues were classified as either marginal (cervical free gingiva), proximal (papillary free gingiva), or mid-gingival (attached gingiva). The size of the abrasions was measured using a periodontal probe (Deppeler) placed across the long axis of the lesions. The lesions were categorized as small $(<3 \mathrm{~mm})$, medium $(3-5 \mathrm{~mm})$, or large $(>5 \mathrm{~mm})$ in all 4 quadrants.

The gingival index was assessed at all visits (baseline, day 7, day 28) of the treatment period, according to the scoring proposed by Löe (Löe H. 1967). The gingival assessment was performed after drying the teeth. Bleeding was provoked by running a blunt pocket probe along the soft tissue wall at the entrance of the gingival crevice.

Assessment always started with the vestibular sites (distal, buccal, mesial) in the maxilla, whereafter the palatinal maxillary surfaces were assessed. The same procedure was performed with the teeth in the mandible (three vestibular and one lingual site). Each of the four sites of each tooth (buccal, mesial, distal and lingual) was assigned a score from 0 to 3 . The sum of the scores divided by the number of investigated sites yields the index for the individual concerned. The average of the four scores per tooth represents the mean gingival index per tooth. 
Plaque index was assessed at all visits (baseline, day 7, and day 28) of the treatment period according to the scoring proposed by Silness and Löe (1964). After drying the teeth with air, the staining agent (Mira-2-Ton ${ }^{\circledR}$ solution, Hager Werken, Duisburg, Germany) was applied. Assessment was performed with a dental probe run along the surfaces. Each of the four tooth surfaces was assigned a score from 0 to 3. Scoring started with the vestibular sites (distal, buccal, and mesial) in the maxilla and continued with the oral (palatinal) surfaces. The same procedure was then performed with mandibular teeth (three vestibular and one lingual site).

\section{Study procedures}

Patients were screened about three days before surgery. At baseline (Visit 1), inclusion and exclusion criteria were re-checked. After the assessment of gingival abrasion, gingival index and plaque index, the subjects underwent the surgical intervention and thereafter received the study products. Brushing started as instructed on the same day. Study parameters, adverse events, and compliance with study procedures were assessed on day 7 (Visit 2) and on day 28 (Visit 3), according to the study schedule.

The study was completed after day 28 and the patients received professional teeth cleaning.

\section{Data analysis}

Statistical comparisons between groups of gingival abrasion, gingival index, and plaque index on day 28 were effectuated by means of an analysis of covariance (ANCOVA) where the baseline values were used as covariates. For the comparisons between groups at Visits 1 and 2, Student's t-test has been used. SAS version 9.1 statistical software was used.

\section{Results}

A total of 173 patients were included in the study, 84 in test group and 89 in control group. All the participants completed the study protocol.

A CONSORT-type diagram (Schulz et al. 2010) explaining the design of this study is presented in figure 3.

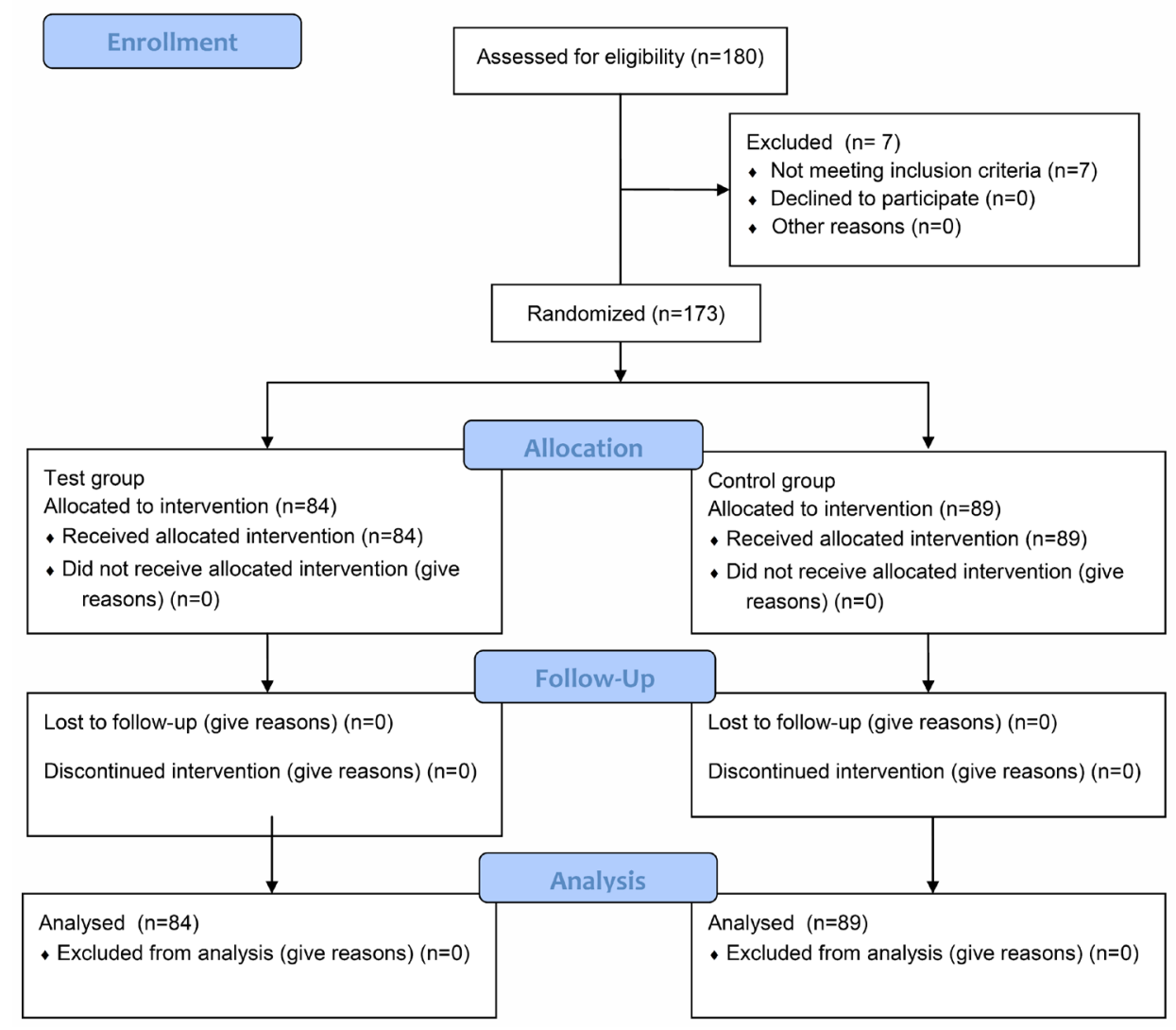

Figure 3. CONSORT-type diagram explaining the design of the study 


\section{Gingival abrasion}

Full details of gingival abrasion are provided in Table 1. Because of the small individual numbers, stratification by lesion size was not suitable for a statistical approach. Table 1 therefore shows the average number of lesions of all sizes for both groups at all three visits. While the average number of lesions was about $45 \%$ higher in the test group at baseline, this figure clearly and consistently decreased in the test group, and it increased in the control group at both later visits. The mean number of lesions of all sizes was after 28 days significantly lower in the test group (p-value $<0.001$ ) compared to control group.

Table 1. Mean number of gingival lesions per patient (mean \pm standard deviation)

\begin{tabular}{|l|l|c|c|c|}
\hline \multicolumn{2}{|c|}{} & ADA (N = 89) & meridol $^{\circledR}(\mathrm{N}=84)$ & $\mathrm{p}$ \\
\hline \multirow{4}{*}{ Small lesions } & Baseline & $0.287 \pm 0.387$ & $0.375 \pm 0.430$ & $0.157^{*}$ \\
\cline { 2 - 5 } & Day 7 & $0.264 \pm 0.354$ & $0.345 \pm 0.360$ & $0.137^{*}$ \\
\cline { 2 - 5 } & Day 28 & $0.368 \pm 0.395$ & $0.161 \pm 0.259$ & $<0.001^{*}$ \\
\hline \multirow{4}{*}{ Medium lesions } & Baseline & $0.132 \pm 0.287$ & $0.238 \pm 0.394$ & $0.046^{*}$ \\
\cline { 2 - 5 } & Day 7 & $0.256 \pm 0.393$ & $0.021 \pm 0.124$ & $<0.001^{*}$ \\
\cline { 2 - 5 } & Day 28 & $0.214 \pm 0.378$ & $0.000 \pm 0.000$ & $<0.001^{*}$ \\
\hline \multirow{4}{*}{ Large lesions } & Baseline & $0.006 \pm 0.053$ & $0.003 \pm 0.027$ & - \\
\cline { 2 - 5 } & Day 7 & $0.039 \pm 0.188$ & $0.012 \pm 0.109$ & - \\
\cline { 2 - 5 } & Day 28 & $0.000 \pm 0.000$ & $0.000 \pm 0.000$ & - \\
\cline { 2 - 5 } & Baseline & $0.424 \pm 0.417$ & $0.616 \pm 0.406$ & $0.003^{*}$ \\
\cline { 2 - 5 } & Day 7 & $0.559 \pm 0.388$ & $0.378 \pm 0.365$ & $0.002^{*}$ \\
\cline { 2 - 5 } & Day 28 & $0.582 \pm 0.382$ & $0.161 \pm 0.259$ & $<0.001^{* *}$ \\
\hline
\end{tabular}

*t test

** Analysis of covariance with baseline values as covariates

\section{Gingival index}

Numerical data are provided in Table 2. The gingival index was slightly lower in the test group at all visits; the difference reached statistical significance on day $7(p=0.021$, t-test). At day 28 the gingival index was significantly lower in the test group ( $\mathrm{p}=0.031$, ANCOVA) compared to control group.

\section{Plaque index}

Numerical data are provided in Table 2. The plaque index was slightly lower in the test group at all visits, but the difference did not reach statistical significance on any one of the visits.

Table 2. Gingival index and plaque index (mean \pm standard deviation)

\begin{tabular}{|l|l|l|l|l|l|c|}
\hline & & \multicolumn{2}{|c|}{ ADA } & \multicolumn{2}{|c|}{ meridol $^{\circledR}$} & $\mathrm{p}$ \\
\hline Gingival index & Baseline & $\mathrm{N}=88$ & $1.212 \pm 0.771$ & $\mathrm{~N}=85$ & $1.072 \pm 0.588$ & \\
\hline & Day 7 & $\mathrm{N}=88$ & $0.706 \pm 0.589$ & $\mathrm{~N}=85$ & $0.528 \pm 0.409$ & $0.021^{*}$ \\
\hline & Day 28 & $\mathrm{N}=87$ & $0.424 \pm 0.433$ & $\mathrm{~N}=84$ & $0.281 \pm 0.299$ & $0.031^{* *}$ \\
\hline Plaque index & Baseline & $\mathrm{N}=88$ & $1.325 \pm 0.799$ & $\mathrm{~N}=85$ & $1.277 \pm 0.723$ & \\
\hline & Day 7 & $\mathrm{N}=88$ & $0.796 \pm 0.550$ & $\mathrm{~N}=85$ & $0.686 \pm 0.391$ & \\
\hline & Day 28 & $\mathrm{N}=87$ & $0.515 \pm 0.416$ & $\mathrm{~N}=84$ & $0.417 \pm 0.307$ & $0.07^{* *}$ \\
\hline
\end{tabular}

*t test

** Analysis of covariance with baseline values as covariates

\section{Adverse events - tolerability}

Adverse events and changes in hard and soft tissues in the oral cavity were recorded and documented at all visits (baseline, day 7, and day 28). No adverse events were reported. 


\section{Discussion}

The bacterial biofilm is commonly held responsible as one main reason for formation of both caries and inflammatory periodontal diseases (Axelsson, Lindhe 1978). Without brushing, plaque accumulates in the subgingival crevices of the teeth and causes gingival inflammation and bleeding (Addy 2008).

Rather the quality of tooth cleaning than the frequency is important for maintenance of oral health (Attin, Hornecker 2005). The use of the hard brush resulted in lower plaque scores and, at the same time, more gingival erosions than the use of the soft brush (Niemi et al. 1984).

The purpose of this study was to compare toothbrushes with differently shaped filaments with respect to cause gingival abrasion after surgical tooth extraction. Secondary endpoints were assessing the impact on general gingival health (gingival index) and plaque removal (plaque index).

The average number of lesions with gingival abrasion per patient slightly and consistently increased over the time in patients using the standard toothbrush with rounded filaments. This figure clearly decreased in patients using the toothbrush with conical filaments. The mean number of lesions of all sizes was after 28 days significantly lower in the test group ( $\mathrm{p}$-value $<0.001$ ) compared to control group. These findings are also supported by the study of Versteeg et al. (Versteeg et al. 2008).

The gingival index was slightly lower in the test group at all visits. At day 28 the gingival index was significantly lower in the test group ( $\mathrm{p}=0.031$, ANCOVA) compared to control group. Similar findings were demonstrated in the study of Sgan-Cohen et al. (Sgan-Cohen, Vered 2005) where the toothbrush with conical filaments showed highly significant statistical decrease of gingival index. This is in contrast with a study conducted by Versteeg et al. (Versteeg et al. 2008), where the toothbrush with conical filaments was less effective in the removal of biofilm and reduction of gingival bleeding than the toothbrush with conical filaments.

Plaque index was not significantly different between the two groups in the last visit. Both toothbrushes showed comparable effectiveness of biofilm removal. This is in contrast to a studies conducted by Dörfer et al. and Checchi et al. (Dörfer et al. 2003; Checchi et al. 2007), in which a groups using toothbrush with conical filaments had a statistically significant reduction in plaque index score to that of a toothbrush with rounded filaments.

Some limitations could be highlighted in this study. Both groups were using the same toothpaste and mouthrinse with amine fluoride and stannous fluoride. This could have influenced the formation of new biofilm and masked the differences between the two toothbrushes in case of plaque index.

This study raised no concerns regarding the safety and tolerability of either toothbrush.

Conclusion: The meridol ${ }^{\circledR}$ special toothbrush with conical filaments compared to the standard ADA toothbrush induced significantly less gingival abrasion and provides effective plaque removal and reduction of gingival index.

\section{Conflict of interest and source of funding statement}

The authors have no conflicts of interest. The study was financially supported by GABA International AG (Therwil, Switzerland).

\section{References:}

1. Addy M. (2008), Oral hygiene products: potential for harm to oral and systemic health? Periodontol, 48, p. 54-65.

2. Attin T., Hornecker E. (2005), Tooth brushing and oral health: how frequently and when should tooth brushing be performed? Oral Health Prev Dent., 3(Suppl. 3), p. 135-140.

3. Axelsson P., Lindhe J. (1978), Effect of controlled oral hygiene procedures on caries and periodontal-disease in adults. J Clin Periodontol , 5, p. 133-151.

4. Daly C.G., Highfield J.E. (1996), Effect of localized experimental gingivitis on early supragingival plaque accumulation. J Clin Periodontol, 23, p. 160-164.

5. Dörfer C.E., von Bethlenfalvy E.R., Kugel B., Pioch T. (2003), Cleaning efficacy of a manual toothbrush with tapered filaments. Oral Health Prev Dent, 1, p. 111-118.

6. Drisko C., Henderson R., Vancey J. (1995), A review of current toothbrushbristle end-rounding studies. Compend Contin Educ Dent, 16, p. 694-707.

7. Checchi L., Montevecchi M., Gatto R.M., Moreschi A., Checchi V. (2007), Clinical efficacy of two toothbrushes with different bristles. Int J Dent Hygiene, 5, p. 242-246.

8. Löe H. (1967), The Gingival Index, the Plaque Index and the Retention Index System. J Periodontol, 38, p. 610-616.

9. Niemi M.L., Sandholm L., Ainamo J. (1984), Frequency of gingival lesions after standardized brushing as related to stiffness of toothbrush and abrasiveness of dentifrice. J Clin Periodontol; 4: p. 254-261. 
10. Page R.C. (1986), Gingivitis. J Clin Periodontol; 13(Suppl. 5), p. 345-359.

11. Saxer U.P., Yankell S.L. (1997), Impact of improved toothbrushes on dental diseases. Quintessence Int; 28: p. 513-525.

12. Sgan-Cohen H.D., Vered Y.A. (2005), Clinical trial of the meridol ${ }^{\circledR}$ toothbrush with conical filaments: evaluation of clinical effectiveness and subjective satisfaction. J Clin Dent; 16: p. 109-113.

13. Schulz K.F., Altman D.G., Moher D. (2010), CONSORT 2010 Statement: updated guidelines for reporting parallel group randomised trials. BMJ, 340: p. 332.

14. Silness J., Löe H. (1964), Periodontal disease in pregnancy. II. Correlation between oral hygiene and periodontal condition. Acta Odontol Scand. 22: p. 121-135.

15. Versteeg P.A., Piscaer M., Rosema N.A.M., Timmerman M.F., van der Velden U., van der Weijden G.A. (2008), Tapered toothbrush filaments in relation to gingival abrasion, removal of plaque and treatment of gingivitis. Int J Dent Hyg, 6: p. 74-82.

16. von Bethlenfalvy E.R., Kugel B., Pioch T., Dörfer C.E. (2002), Einfluss unterschiedlicher Filamente auf die Gingivitisreduktion zweier Handzahnbürsten. Parodontologie, 13, p. 286-287.

Submitted: 02.12 .2014

Accepted: 26.02 .15 